\title{
Can (quantum) information be sorted out from quantum mechanics?
}

\author{
Michele Caponigro $^{1}$ and Stefano Mancini ${ }^{2}$ \\ ${ }^{1}$ Department of Epistemology of Complexity, \\ University of Bergamo, 24100 Bergamo, Italy, EU \\ ${ }^{2}$ Physics Department, University of Camerino, \\ 62032 Camerino, Italy, EU
}

November 16, 2009

\begin{abstract}
We shall draw an affirmative answer to the question posed in the title. The key point will be a quantum description of physical reality. Once fixed at ontic level two basic elements, namely the laws of physics and the matter, we argue that the underlying physical reality emerges from the interconnection between these two elements. We consider any physical process, including measurement, modeled by unitary evolution. In this context, we will deduce quantum randomness as a consequence of inclusion of the observer into the quantum system. The global picture of the universe is in a sense deterministic, but from our own local perspective (as part of the system) we perceive quantum mechanical randomness. Then, the notion of "information" turns out to be a derivative concept.
\end{abstract}

I've spent much more time thinking about the photon problem [i.e., about quantum theory] than about relativity. A. Einstein 


\section{Introduction}

The abstract mathematical structure of the Lorentz transformations was deduced through simple physical principles. Thanks to the existence of these physical principles we do not have a significant debate on the interpretation of the theory of special relativity. The formulation of Quantum Mechanics (QM), to the contrary, is based on a number of rather abstract axioms without a clear motivation for their existence. Despite its success, the absence of elementary physical principles has determined a broad discussion about the interpretation of the theory. For this reason, and not only, Bell called the ordinary QM with the abbreviation FAPP (for all practical purposes).

Over the years there has been a permanent wish to have a realistic view of the Nature also in the quantum framework. According to de Muynck [10], we can distinguish a realist interpretation of QM thought to describe microscopic reality (likewise classical mechanics is generally thought to describe macroscopic reality), from an empiricist interpretation where state vector and density operator are thought to correspond to preparation procedures, and quantum mechanical observables correspond to measurement procedures.

Recently, with the development of quantum information theory, several scientists have given to information a fundamental role in the description of Nature. All these approaches (quantum theoretic description of physical systems) start in general from the assumption that we live in a world in which there are certain constraints on the acquisition, representation, and communication of information. Several works claim that QM can be viewed as information theory $[3,4,7,11,14]$. According to them, the description of physical systems in terms of information and information processing is complementary to the conventional description of physical systems in terms of the laws of physics (or the only way to describe them). In Ref.[3] it was sentenced: "The notion of quantum information is to be understood as a new physical primitive." That is, the laws of physics are reducible to information.

The primitive role of information seems to explain, according to some authors, the deep nature of physical reality. In this context, the description of a state of a quantum system is a description of the information possessed by the observer about the system (in this case, the measurement is not a physical process). The quantum state is a construct of the observer and not an objective property of the physical system. In particular, in Ref.[7] it was claimed that the nature of reality can be explained by subjective knowledge.

Recently, moving from the renowned Landauer's statement [8] "Informa- 
tion is Physical" we are witnessing a drift towards the statement "Physics is Informational". Here we would supply some reasonings against that.

We start our analysis by introducing essential hypothesis to infer the possible underlying physical reality. These hypothesis will be based on considerations of physical nature. We will assume that any consistent description of Nature is a sort of "isomorphism" between the laws of Nature and the matter. With the term isomorphism we intend that the theoretical structure of physical law is consistent with physical phenomena. A physical law has a mathematical structure, but a mathematical structure is not a physical law. The structure alone cannot predict physical behavior of Nature, we need of physical and mathematical axioms together. The physical axioms are determined by empirical facts, which in turn require the presence of the objects. Objects interact each other, hence any physical process, including measurement, should be modeled by unitary evolution. As matter of fact objects are also observes. Then, quantum randomness is a consequence of inclusion of the observer into the quantum system. From that, it follows the concept of information. This approach leads us to conclude that the information has not the statute of physical law and it is reducible to the laws of physics, i.e. it is a derivative concept. The layout of the paper follows the scheme of Fig.1. In Section II we discuss about laws of physics and matter. Section III is dedicated to the relation between observers and physical objects. In Section IV quantum correlations are invoked to describe quantum measurements by means of unitary evolution. Then, Section V deals with the origin of quantum randomness. Finally, in Section VI (quantum) information is explained as a derivative concept (coming from laws of physics and matter). Section VII is for conclusions.

\section{Laws of physics and matter}

We start by considering three elements: (i) physical laws, (ii) information and (iii) matter. Our main objective is to find a possible link among them. We will argue that the physical reality is a structure emerging from an "isomorphic" relation between physical laws and matter. These will be primitive concepts with respect to information.

From the philosophical point of view it could be interesting to analyze various combinations of the above three elements. In general we can say the following: if we take the laws of physics at ontic level, we have a form 


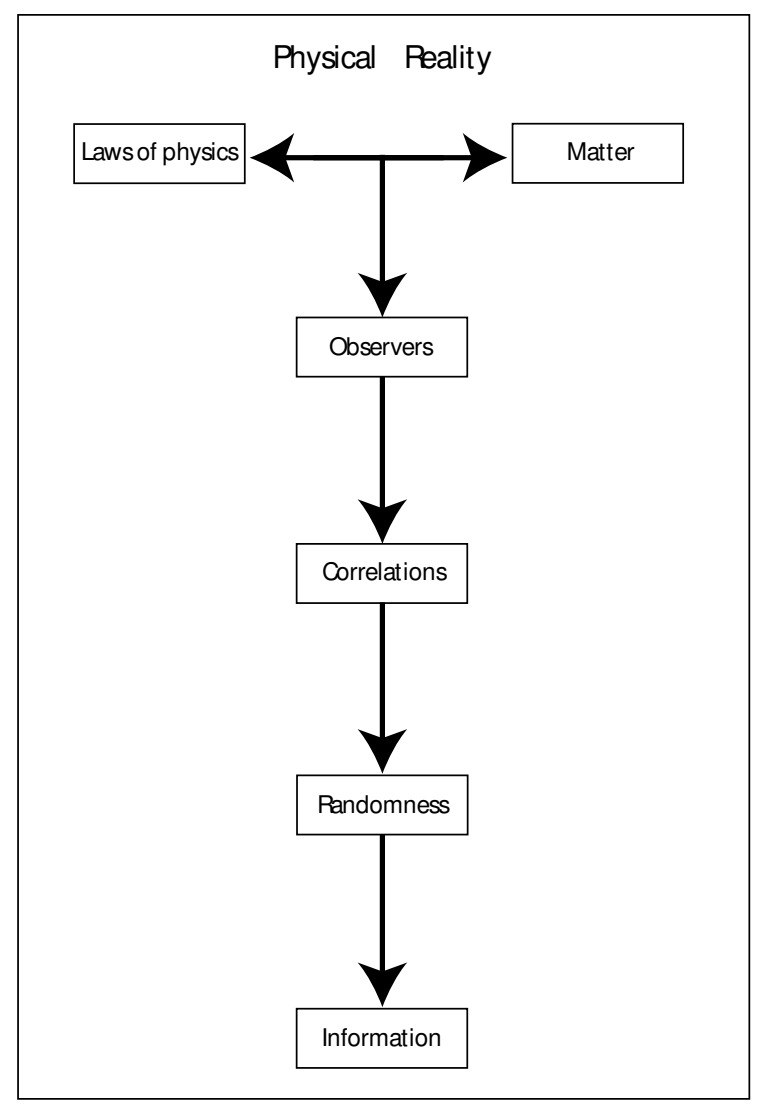

Figure 1: A scheme summarizing the used reasoning.

of Platonism; if we take the information at ontic level we have to do with idealistic forms, while we have realistic forms if we take the matter at ontic level. In the Platonic view, the dependence among the above three elements is: laws of physics $\Rightarrow$ matter $\Rightarrow$ information.

We do not join the full platonism, where the perfect laws of physics (which live elsewhere) can be computed to arbitrary precision with the unlimited amounts of information (in this case the Platonic realm is the "real reality"). We admit the importance of mathematical structure of a physical law, but we must combine it with the physical constraints, which are determined by empirical facts, i.e. the correspondence with the matter. 
Physics is the study of the behavior of physical objects ${ }^{1}$ located in a space. Thus, the objects are governed by the space's geometry. A fundamental aspect of a geometry is the group of transformations defined over it. Then, the field of numbers is crucial to describe essential properties of the geometry.

Physics is that take place in space and our geometry is that physics takes place within in. It is not possible to think physics without space. On the converse it is possible to think to geometry, many geometries, without physics. Physics and the geometry in which it takes place are not independent. There is a close relation between them [9]. Moreover, group theory is the necessary instruments for expressing the laws of physics (the concept of symmetry is derived from group theory). The invariance under the group of transformations is a fundamental criterion to classify mathematical structures.

We assume that physics describes the behavior of many objects, that they affect each other, i.e. they interact each other, thus change each other. All physical objects, assumed to be interacting, must be described by functions (of both space and time). They cannot be simply described by variables (functions of time alone). These functions of (of space and time) must be group-representation basis states, actually Poincaré group representation basis-states, thus complex. This is because a fundamental fact about our Universe is the invariance of the laws of physics under the Poincaré group.

It then follows that QM and relativity are both consequences of geometry and the necessity for interactions [9] (in the most economic way according to Occam's razor). Thus Schrödinger equation is not and independent assumption, but rather direct requirement of the Poincaré group, through the Dirac equation in the appropriate limit.

\section{$3 \quad$ Observers and objects}

In QM it was introduced the fundamental notion of "observable". Is this term related with measurement or with an experimental process? The observable notion need of the observer, hence it is responsible for the division of the world in two parts: physical system and apparatus. While the notion of "measurement of the observable" requires the measurer (observer), the notion of "experimental observation process" has to do with results and facts. Our thesis is that QM has to do with laws and experimental results. In

\footnotetext{
${ }^{1}$ We cannot specify further the emaning of this term unless using a tautology. We simply consider physical objects as elements of matter.
} 
this framework, the measurer can be considered like an object being part of the system (in the same way it can be considered measured/observed). All physical objects are observers, and all observers are physical objects, and because all observations are interactions and all interactions are observations, all physical objects and all physical processes should be governed at all times by the basic equations of QM, or more general by the transformations of the Poincaré group, from which these equations follow.

We list some things supporting the inclusion of the observer into the system.

- The apparatus (the observer) should not be separated off from the rest of the world into black boxes, as if it was not made of atoms and not ruled by QM.

- We have not a theory of the measurer, we do not know who is the measurer and when it is authorized to make a measure.

- We can easily interpret the notion of "experimental results". Within a result (fact) of an experimental process, we have both the measurer and the system. An experimental fact has two qualities: to include the observer in the system (without any privileged role) and to give us empirical results.

- An empirical fact is interpreted as interaction between objects governed by physical law.

In the light of these considerations, we argue that everything we know about physics is compatible with the hypothesis that all physical processes, including measurements, can be accurately modeled by unitary evolution of the wave function. This view seem to led us to consider some features ascribed to many worlds interpretation of the quantum theory [6].

According to this interpretation, the world we live in is continually branching into multiple near-copies corresponding to different possible measurement outcomes. Unitary quantum dynamical laws describe the evolution of all these branches simultaneously. The definite measurement records that we observe, remember and communicate, are just characteristics of individual branches. Then, the development of all quantum systems are governed by the same unitary dynamical laws and hence develop completely deterministically and linearly. In this context, the wavefunction describes real properties, 
so that all speculations about determinism, causality, quantum jumps and collapse of wavefunction are unnecessary.

When a microscopic quantum system interacts with a macroscopic apparatus, decoherence drives the "collapse" of the wave function (FAPP). All possible outcomes of any measurement are regarded as real but we perceive only a specific outcome, because the state of the observer as part of the quantum system is strongly correlated with that outcome. In this context, the evolution of the wave function is deterministic, however we are unable to predict with certainty the outcome of an experiment to be performed in the future. We do not know what branch of the wavefunction we will end up, so we are unable to predict our future state. Thus, while the global picture of the universe is deterministic, from our local perspective (within the system) we perceive quantum mechanical randomness.

The Universe is governed by QM. So a system, an electron, an atom, a molecule, a crystal, a person, the universe itself is described by a statefunction. There is not distinction between observers and physical objects, they are the same set of things, meaning that the laws of QM must apply to all observers (including human beings). Statefunctions of an object are defined, can only be defined, with respect to another physical one: they are given relative to a frame of reference. A frame of reference is a class that can contain a physical object. We have to do with a set of observations of one physical object by another.

Once established these basic points we should focus on the mathematical tool already available in the quantum theory which is able to preserve the above prescriptions, specifically the concept of relative state, and quantum correlations (entanglement).

\section{Quantum correlations}

The meaning given to an entangled state by the many-worlds interpretation [6] could add new elements useful in our analysis. According to this interpretation, the terms of an entangled state describe something that really exist; the state does not just refer to the probabilities of results that would be obtained if measurement takes place. The different terms of an entangled state can be interpreted as showing that the universe branches into a number of different worlds. What are really important are the correlations (this is also in line with the relational approach to quantum mechanics [12]). The main 
ingredient is thus the relative state.

Let us say that an observer $O$ is going to perform a measure of the observable $B$ on the system $S$ being in a superposition state: $|\mathbf{S}\rangle=\alpha\left|\varphi_{B}\right\rangle+$ $\beta\left|\phi_{B}\right\rangle$; where $\left|\varphi_{B}\right\rangle$ and $\left|\phi_{B}\right\rangle$ are eigenstates of $B$. Before the measurement is performed, the state of the composite system (Observer plus System) is

$$
|\mathbf{O}+\mathbf{S}\rangle^{0}=|\operatorname{Ready}\rangle_{O}\left(\alpha\left|\varphi_{B}\right\rangle_{S}+\beta\left|\phi_{B}\right\rangle_{S}\right) .
$$

After the measurement (according to Shrödinger equation evolution) the composite system will be in a state

$$
|\mathbf{O}+\mathbf{S}\rangle^{1}=\alpha\left|\varphi_{B}\right\rangle_{O}\left|\varphi_{B}\right\rangle_{S}+\beta\left|\phi_{B}\right\rangle_{O}\left|\phi_{B}\right\rangle_{S}
$$

where the observer results entangled with the observed system.

The physical meaning, according to this interpretation, relies on the correlations. Each component of the wave function is called branch, and the branching is responsible for our experiences. These are the consequences of the fact that there is not interaction between branches, but every subsystem can only "see" the other subsystems states that are in the same branch. In this way, the quantum "world" is always decomposable into system and observer. The key idea is that their correlations defines a preferred set of basis vectors.

The relevance of quantum correlations has been stressed also in Ref.[1]. There, it was claimed that only correlations, not the correlata of a quantum system, are physically accessible, but we have to include the observer as one of its parts. As a consequence, quantum reality is "real" in the sense that QM completely and deterministically describes the evolution of a closed system (not just its wavefunction), and that the statistical character arises from the fact that an observer, because he is part of the closed system, is offered an incomplete view of the quantum system he attempts to measure. Therefore, the quantum universe is deterministic as Einstein's physical reality demands, but must include the observer as one of its parts due to the inseparability of entangled quantum states.

\section{Quantum randomness}

We have established that the inclusion of the observer into the system is responsible of quantum randomness. We now analyze two approaches that 
tries to interpret the source of the randomness: an informational approach [7] and a tomographic approach [5].

In Ref.[7], the quantum state does not represent information, it represents an individual agent's subjective degrees of belief about what will happen in a measurement. The concept of information, here is not considered as "objective" data to acquire. The information is linked with the subjectobserver and depend from the choice and belief of the observer. The structure of this interesting position is centered on the interpretation of probability: the Bayesian interpretation.

A first possible criticism to this approach is: how to demonstrate that information belongs to the subject? Is it possible to assume that information is related to the system?

The central role played by Bayes theorem relies on the possibility to reverse the conditional probability. In a quantum context, suppose that $\left(\Psi_{0}\right)$ is the preparation of a particular quantum state, and $\left(\Psi_{B}\right)$ is a particular outcome of a measurement of the state. To infer how the state was prepared, we need to compute $p\left(\Psi_{0} \mid \Psi_{B}\right)$, but we only have $p\left(\Psi_{B} \mid \Psi_{0}\right)$. So we have to make a guess about $p\left(\Psi_{i}\right)$ (we are doing assumptions on the preparation of the state), e.g. by adopting the "principle of indifference" for which $p\left(\Psi_{i}\right)=$ $p\left(\Psi_{j}\right), \forall i, j$. Then

$$
p\left(\Psi_{B}\right)=\sum_{i} p\left(\Psi_{B} \mid \Psi_{i}\right) p\left(\Psi_{i}\right),
$$

and by applying the Bayes' rule

$$
p\left(\Psi_{0} \mid \Psi_{B}\right)=\frac{p\left(\Psi_{B} \mid \Psi_{0}\right) p\left(\Psi_{0}\right)}{p\left(\Psi_{B}\right)} .
$$

The prior probability is the fundamental point between a possible subjective or objective interpretation of Bayes' theorem. In order to recover an objective theory, we must interpret probability in QM not as prediction based on our actual state of knowledge but rather as a prediction based on the most complete possible knowledge about quantum state. A prediction based not on what we know, but on the prediction that anyone can make, no matter how much they know. It is in this sense that the outcome is truly random: it cannot be predicted with certainty even when our knowledge is complete. Truly randomness is a consequences, according our hypothesis of the observer being part of the system and the basic role of correlations in a quantum system. 
Probabilities arise because we (as part of the system) cannot predict our future with certainty, so while the wave function of the universe is deterministic we can do no better than making probabilistic predictions.

Then, we summarize below our criticisms to the informational approach:

- An unknown quantum state is unknowable, but an unknown state does not mean that we have to do with a subjective state. There are not an automatic link between unknown state and subjective state. In this way, we are replacing the unknown state with our knowledge of the same state (epistemic role).

- Informational approach considers information at ontic level. As a consequence the observer is an external object that acquires information from a random world. In this way he builds his own subjective world of beliefs.

- It is not possible to build an ontology of QM. Quantum system are defined by attributes, such as position, momentum, angular momentum, and energy or Hamiltonian. These attributes and thus the numerical particulars of their eigenvalues and eigenfunctions and their inner products are objective properties of the system. A Bayesianist intends the value assumed by an attribute not as an objective property, and the quantum state that we use to describe the system as purely subjective.

The tomographic approach has the main objective of inferring (in indirect way) a quantum state [5]. In this context, we will ascribe at the probability an ontic role. In fact, in a tomographic framework, we are like the prisoners in Plato's famous parable who were chained in a cave and forced to see only the shadows of the things outside but not the things as they are. Our epistemological path is in syntony with the analogy of Plato's cave, because we have considered the observer as part of the system and his statistical description of the physical reality (shadows) is a consequence of his inclusion.

We must pay attention to not confuse the accessibility of underlying physical reality and the subjective knowledge of the same reality, in other words between information and the existence of the physical reality.

The tomographic approach relies on the possibility to characterize a system (either quantum or classical) by a set of fair probability distributions. 
Such probabilities (marginals or tomograms) depend upon a stochastic variable and some parameters. They represent the shadows a system may have in "directions" specified by the values of parameters. By means of these shadows we can infer the reality (reconstruct the state).

Actually, the tomographic approach provides a unifying framework where quantum and classical aspects could be better compared. So that the interpretational problems are moved to the probability field, and go back to the debate on the nature of probability (subjective or objective character of probability). Again, to have an objective theory we must interpret probabilities not as prediction based on our actual state of knowledge, but rather as prediction based on the most complete possible knowledge of the quantum state (in contrast to pseudo-randomness that arises in classical mechanics because of incomplete knowledge). As matter of fact, the information contained in the marginal distributions is complete. An ontic state can be represented through classic approach by the marginal probability distributions and is characterized by the fact that there are no other marginal probability distribution with the same collection of actualized observables. We retain the one-to-one correspondence between the ontic state of an object and this mathematical object.

The tomographic approach to quantum mechanics is a classic-like approach, but the probability inferred is not similar with classic epistemic probability. We argue that our assumptions bring us to establish an ontological probability of the physical reality described by classic tomography. Thus our tomograms assume an ontological character and since they completely characterize the state of a system, also the latter assumes such a character. QM does not force us to give up realism, but it force us to distinguish carefully between potential and actualized properties.

\section{6 (Quantum) Information}

The philosophical questions we would address here are: what information is and what is its role in physics ? Laws of physics is just a representation of information or information is a representation of physical law? Would it be natural to suppose that there are information laws not less real than physical laws?

We utilize in our analysis the general concept of information as defined in Shannon's theory [13]. "Information in communication theory relates not 
to what you do say, but to what you could say". Hence, information is related to many possibilities, different choices and distinguishability. Actually, information emerges from different possible configuration for objects.

Why to include quantum systems? Because, the theory does not place restrictions on the types of physical systems that can be used in a communication system. The theory does not require classical systems, these facts suggest that a new concept of information is not required. ${ }^{2}$

Information is related to at least two physical systems. For instance, a communication scheme presupposes a source and a destination of information, thus implying that information describes relational properties of physical systems. When we speak about the information contained is a system, we implicitly speak about a relational properties of that system to other systems. Hence, information is not a physical quantity or absolute property of a system, because it does not determine any properties of a system. However, we should not confuse information with the property of a physical system identified by the Shannon measure.

For these reasons information is reducible to a physical theory and not vice versa. Information remains an abstract, rather than a concrete, noun. That is, information is a derivative concept, there is no information without physical representation (i.e. physical objects and physical laws). If we refer to Landauer [8] "information is exclusively stored in the configuration of physical objects, and transmitted only by material entities". In this way, with tacit conditions, Landauer assumed the presence of matter and laws of physics as primitive. Information then assumes a relational character, moreover it is linked to the concept of many possibilities and as consequence to uncertainty. Information is anchored to the concept of probability which assume in the same quantum context an intrinsically objective character. The relational character of the information and objective intrinsic probability can be explained with the assumption of the inclusion of the observer as part of the system.

\footnotetext{
${ }^{2}$ In the light of quantum information some authors [2] disagree with this position. They claim that quantum information is something radically different from Shannon information. Others [7] claim that Shannon concept of information is appropriate for quantum information as well. We assume the latter position because quantum information theory, although identifies new physical resource (qubits, entanglement), does not introduce a new concept of information.
} 


\section{Conclusion}

We have discussed about a possible description of quantum physical reality based on qualitative assumptions. We have fixed at ontic level two basic elements: laws of physics and matter. We have argued that the underlying physical reality emerges from the interconnection between these two elements and that the information has not a primary role in the description of physical reality. The conditions that permit information to emerge from the underlying physical reality are the existence of an "object" with a multiplicity of possible events (different possible configurations) and the existence of an "object" which acquires it. Starting from this qualitative analysis, we can see that information is a derivative concept whose existence is subordinate to the relationship between more fundamental notions such as objects located in a space (matter) and mathematical tools to characterize it (physical laws). At the origin of ambiguities is the famous Landauer's statement [8]: "Information is Physical", which is too often reversed into "Physics is Informational". Landauer's statement clearly reveals that information is encoded on physical supports (objects, matter) and processed by physical operations subordinate to physical laws. Therefore, matter and physical laws have a primitive character, while information has a derivative character (as supported by Landauer himself). This brings us to devise a logical pathway which supports the above conclusions and describes how the concept of information derives from quantum physics (not vice versa). The merit of quantum physics has been to highlight the role of the observer, but we do a mistake in identifying the observer with a human being, that is giving a privileged role to the observer (and moreover external to the system). The observer/observed role is typical of any physical objects and relies on interaction between objects. In this way, the observer loses the property of being a privileged reference point. In this framework information assumes a purely relational character. In addition, it is linked to the idea of different possibilities through the uncertainty as consequence of the quantum probability, which has an intrinsic objective aspect. Thus, the relational character of the information and the intrinsic objective probability are explained by the fact that the observer is within the system. The objects that can play the role of observer and observed are actually quantum correlated subsystems of a physical system. The entangled states cause a statistically objective and incomplete view of physical reality.

To conclude, we can say that quantum reality is "real" in the sense that quantum mechanics describe completely and deterministically the evolution 
of a closed system and the statistical nature arises from the inclusion of the observer as part of the system. Within this framework one is naturally led to consider (quantum) information as as a derivative concept, that is one can sort it out from quantum mechanics.

\section{References}

[1] Adami C. and Cerf N. J., What Information Theory Can Tell Us About Quantum Reality, arXiv:quant-ph/9806047v1.

[2] Brukner C. and Zeilinger A., Conceptual inadequacy of the Shannon information in quantum measurements, Physical Review A (2001) 63, 022113.

[3] Bub J., Quantum Mechanics is About Quantum Information, arXiv:quant-ph/0408020v2.

[4] Bub J., Clifton R. and Halvorson H., Characterizing Quantum Theory in Terms of Information-Theoretic Constraints, Foundations of Physics (2003) 33, 1561.

[5] Caponigro M., Mancini S. and Man'ko V. I., A Probabilistic Approach to Quantum Mechanics Based on Tomograms, Fortschritte der Physik (2006) $\mathbf{5 4 , 6 0 2 . ~}$

[6] Everett III H., Relative State Formulation of Quantum Mechanics, Review Modern Physics (1957) 29, 454.

[7] Fuchs C., Quantum Mechanics as Quantum Information (and only a little more), arXiv:quant-ph/0205039v1.

[8] Landauer R., The Physical Nature of Information, Physics Letters A (1996) 217, 188.

[9] Mirman R., Group Theoretical Foundations of Quantum Mechanics, Nova Science Publishers (1995), Commack NY.

[10] de Muynck W. M., From Copenhagen to Neo-Copenhagen Interpretation, arXiv:0709.2613v1. 
[11] Parwani R. R., Information Measures for Inferring Quantum Mechanics, Journal of Physics A (2005) 38, 6231.

[12] Rovelli C., Relational Quantum Mechanics, International Journal of Theoretical Physics (1996) 35, 1637.

[13] Shannon C. E. and Weaver W. W., The Mathematical Theory of Communication, University of Illinois Press (1949), Urbana IL.

[14] Zeilinger A., The Message of the Quantum?, Nature (2005) 438, 743. 